\title{
Stand-by spillways in phreatic dams
}

\author{
Andrey Mikhasek ${ }^{1, *}$, Maxim Rodionov ${ }^{1}$, Nikolay Malyugin ${ }^{1}$, and Artem Klimov ${ }^{1}$ \\ ${ }^{1}$ Samara State Technical University, Academy of Architecture and Civil Engineering, \\ Molodogvardeyskaya St., 194, Samara, 443001, Russia
}

\begin{abstract}
Hydraulic structures operation is always connected with accident risks. The authors analyzed emergency situations which affected dams located on the territory of the USA and Russia. This analysis showed that the most dangerous accident which took place at hydroelectric complexes is an overfall through the crest of the dam accompanied with the formation of washouts and blow-out waves. The most striking example here is the accident at St. Francis dam. According to most researchers, its main cause was the human factor. To reduce economic losses which are due to an accident on the hydro-technical construction and to reduce the risk of human influence, the authors propose to use a stand-by spillway in the body of a phreatic dam. For this purpose, they introduce two constructions. The first construction has a melting insert and a spillway channel fastening made of synthetic materials. The second one has filtering covering of a crest and a downstream slope made of coarse-pored concrete. The researchers perform physical and numerical modeling of the developed structures to compare them. On the basis of laboratory experiments and calculations in the program complex, they obtain discharge coefficient values for various spillways, which can be later used for calculating spillway capacity as well as for its construction.
\end{abstract}

\section{Introduction}

The destruction of the phreatic dam is one of the most dangerous accidents at hydroelectric complexes leading to significant economic, environmental and social consequences. They also affect biological resources of the tail water. On 15.000 large dams in the world [1] an average of one and a half cases of destruction occurred per year. The most frequent episode of the dam failure is the formation of the washout in the phreatic dam (up to $80 \%$ of catastrophes that have occurred).

On March 12, 1928, an accident occurred at the 59-meter high St. Francis dam. The water rushed down the canyon up to 40 meters and washed away the power plant located $25 \mathrm{~km}$ down the river. The valley was drowned for $80 \mathrm{~km}$. Not all residents who found themselves in the accident zone were rescued. Approximately 600 people died. The cause of this catastrophe was insufficient attention of engineers who missed the appearance of major cracks in the concrete dam under construction. The destruction of this large project is a striking example of human error on choosing a design solution [2].

\footnotetext{
* Corresponding author: andremixas@mail.ru
} 
The development of modern knowledge is on the way of reducing risks and human errors when ensuring the minimum resource consumption. We see the solution of this problem in achieving the reliability of hydraulic projects, i.e. construction of stand-by spillways in the body of phreatic dams.

\section{Materials and Methods}

The analysis of known solutions of stand-by spillways in the body of phreatic dams [3] made it possible to distinguish two principal decisions:

- the spillway with the melting insert, e.g. the hydroelectric complex Eppalac in Austria and Box Bute in the USA;

- the filtering spillway arranged on top of the crest and downstream slope.

The spillway with a melting insert proposed by us differs from the known solutions in that the melting insert, overflow face and tail water channel are from geosynthetic materials and form the step-curvilinear outline (Figure 1, a).

A distinctive feature of the filtering stand-by spillway design is the absence of the melting insert. Instead of it we suggest that the filter layer should be laid in the upper part of the dam and the downstream slope. For creating a filter spillway we recommend rock materials, e.g. mass concrete or asphalt concrete with a high mass concrete content. It has a number of advantages over stand-by spillways with a melting insert. They are as follows:

- reduction of tail erosion;

- these materials create a protective coating for heaving soils;

- they are the element of slope protection;

- they allow water flow to pass through the crest, if necessary;

- there is no need to lower the dam crest level;

- the possibility to pass across the dam crest during water discharging.

Disadvantages include:

- low discharge capacity;

- there are no recommendations on the design and construction of similar spillways.

To prove the design dimensions of spillways with a melting insert made of synthetic materials and filter spillways, it is proposed to use the dependence of the following type:

$$
Q=m \times B \times \varepsilon \times \sigma \times(2 g)^{1 / 2} \times H^{3 / 2}
$$

$Q$ - design flow; $m$ - flow coefficient; $B$ - spillway width; $\varepsilon$ - coefficient of lateral compression; $\sigma$-coefficient of flooding; $H$ - overflow height.

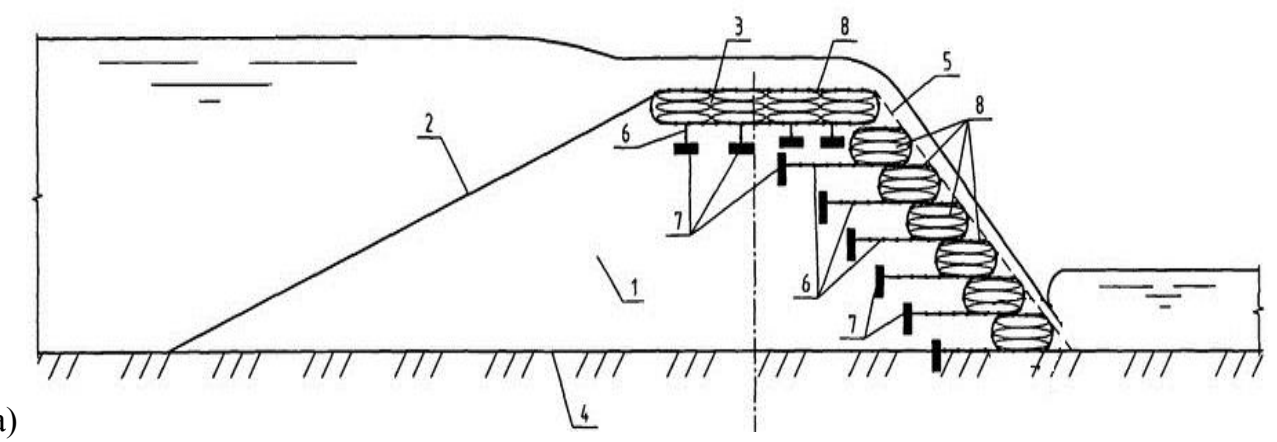


b)

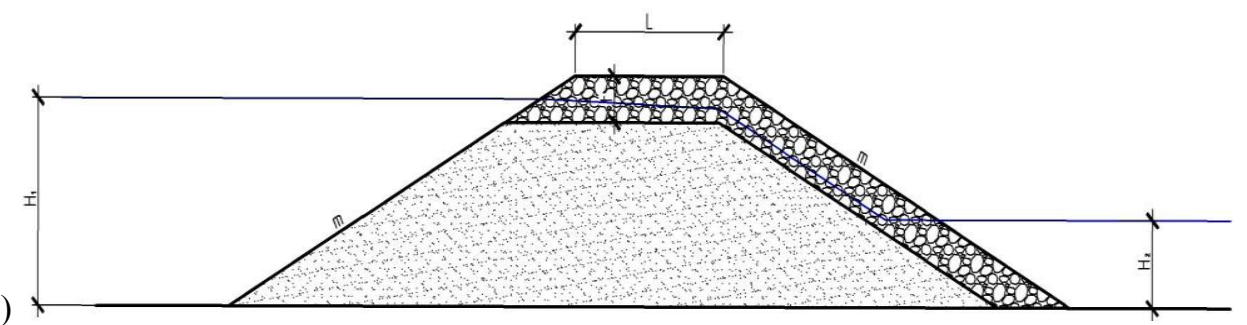

Fig. 1. The design of the stand-by spillway: a. width the melting insert and step-curvilinear downstream slope; $b$. with the filtering insert; 1 - body of the overflow earth dam; 2 - upstream slope; 3 - lip; 4 - foot of the dam; 5 - downstream slope; 6 - reinforcing net; 7 - anchor units; 8 - elements of fastening from flexible envelopes.

To obtain data on the discharge rate coefficients $(m)$ of the developed types of stand-by spillways, the authors carried out physical and numerical simulations. The physical simulation was performed at the hydraulic flume in the laboratory of the Department of Environmental and Hydraulic Engineering ASA SamGTU (Figure 2). The numerical simulation was carried out in the version 17.2 of the ANSYS package. The CFX block was used as a solver.

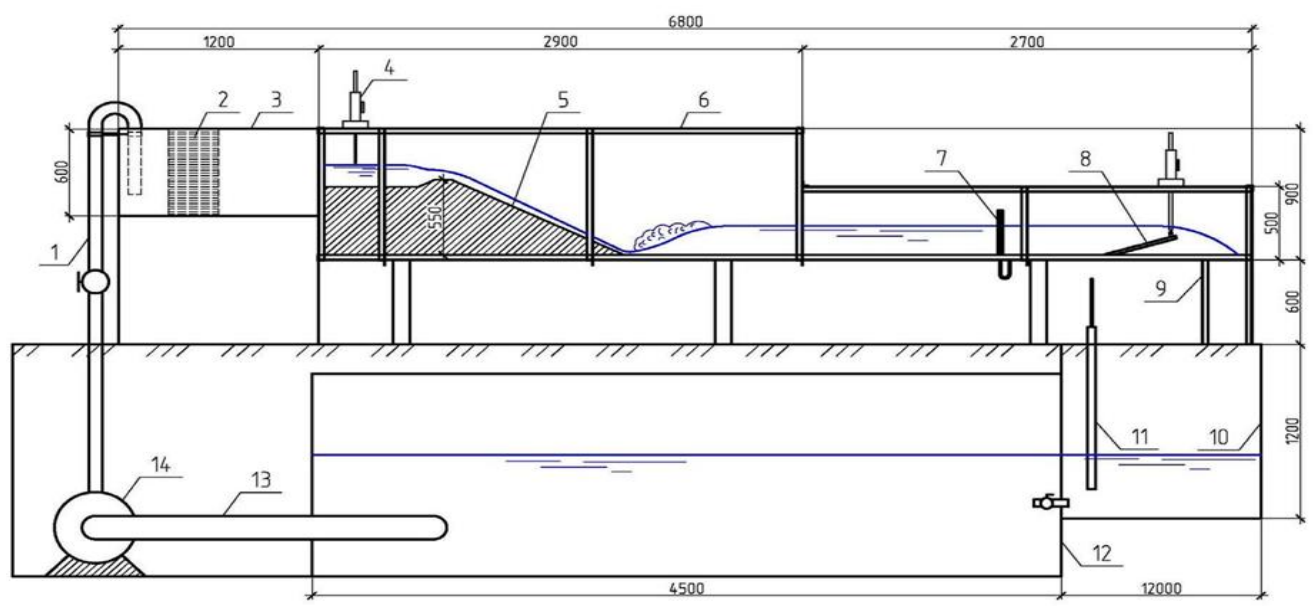

Fig. 2. The design of the experimental plant: 1 - head race; 2 - head damper; 3 - stilling tank; 4-stechpegel; 5 - model under study; 6 - flow section; 7 - piezometer; 8 - rotary gate; 9 - discharge water conduct; 10 - measuring tank; 11 - measuring piezometer; 12 - storage container; 13 - water line to the pump; 14 - centrifugal type pump. Dimensions are given in $\mathrm{mm}$.

The stand-by spillway model with a step-curved downstream side was made from colored gypsum elements of the curvilinear cross section fastened to the plywood sheet. Experiments were carried out for the coefficient of the downstream slope which equals 3 . When performing numeral simulation of the filtering spillway operation, the $20-40 \mathrm{~mm}$ stone concrete was considered as a filtering element.

\section{Results}

Results of the laboratory experiments carried out on the physical model of the stand-by spillway with a melting insert and step-curved downstream side are given in Table 1. 
Table 1. Results of hydraulic investigations of the spillway with a melting insert

\begin{tabular}{|c|c|c|c|}
\hline$Q, \mathrm{~m}^{3} / \mathrm{s}$ & $H, \mathrm{~m}$ & $b, \mathrm{~m}$ & $m$ \\
\hline 0.00313 & 0.038 & 0.23 & 0.412 \\
\hline 0.00444 & 0.048 & 0.23 & 0.414 \\
\hline 0.00645 & 0.063 & 0.23 & 0.401 \\
\hline
\end{tabular}

In Table 1: $Q$ - rate of discharge in the hydraulic flume; $H$ - water height at the crest; $b$ - width of the hydraulic flume; $m$ - experimental coefficient of the discharge.

Results of the numerical simulation of the filtering stand-by spillway are given in figure 3 .

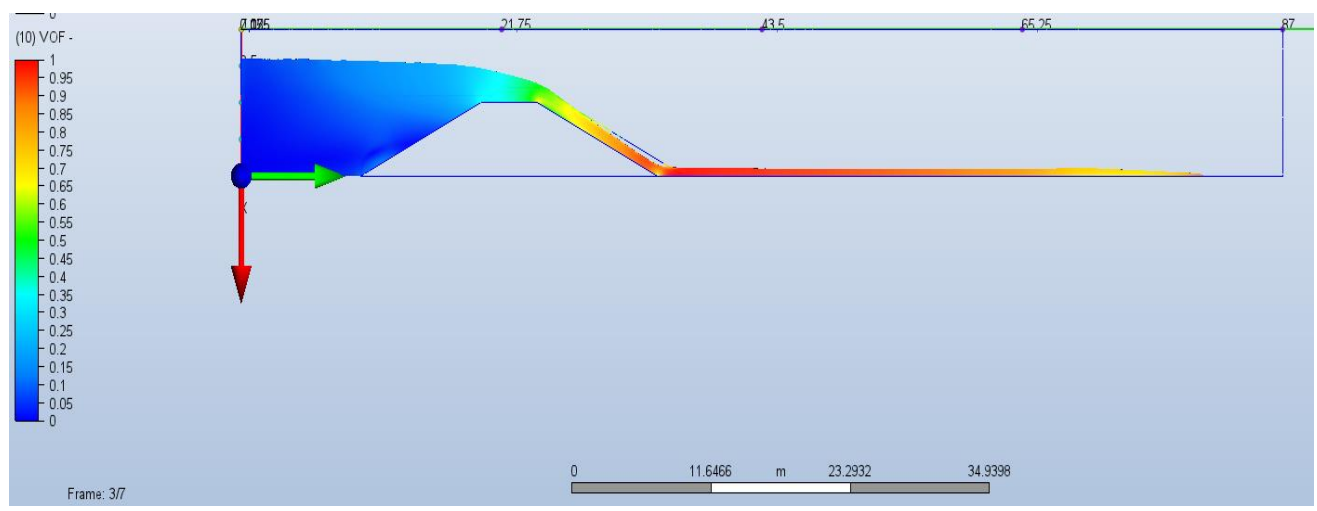

Fig. 3. Results of the numerical simulation of the filtering stand-by spillway

\section{Discussion}

Designs of stand-by spillways in the body of phreatic dams, described in the paper, have certain engineering aspects which do not allow the use of the known reference data, in particular, the data on spillway flow coefficients.

Studies results of spillways with a melting insert and step-curved downstream side showed that the obtained values of flow coefficients are somewhat larger than for the trapezoidal spillway with the same parameters and a smooth downstream side. According to [4], the flow coefficient for the latter is $m=0.38$. Thus, values of flow coefficients for the step-curved downstream side depended on the flux motion regime in the spillway. At low discharge rates we observe step-by-step regime and flow coefficient values are close to $m=0.41$. At the increased discharge rates and appearance of transition and sliding regimes the flow coefficient decreases to $\mathrm{m}=0.40$. The laboratory's capabilities failed to obtain its boundary values at the sliding regime.

\section{Conclusions}

The research yielded the following conclusions:

1. The development of modern knowledge is on the way of reducing risks and human errors when ensuring the minimum resource consumption both during construction and operation. To solve this problem to ensure the reliability of hydraulic facilities, it is necessary to construct stand-by spillways in phreatic dams.

2. The influence of stand-by spillway design features in calculating its discharge capacity is determined by the flow coefficient value $\mathrm{m}$. 
3. In calculations $m$ the values for spillways with a melting insert for a step-curved side $1: 3$ are recommended to be taken not less than 0.4 .

\section{References}

1. A. E. Asarin, Hydraulic engineering, 4, 37-41 (2001)

2. I. A. Alvi Human Factors in Dam Failures. ASDSO Annual Conference. Providence: Association of State Dam Safety Officials (2013)

3. Yu. M. Kosichenko, E.D. Mikhailov, Scientific review of the Institute of Irrigation, 2, 124-137 (2014)

4. P. G. Kiselev, Hydraulic design reference guide (Energy, Moscow, 1972) 